\title{
Consensus Clustering for Microarray Gene Expression Data
}

\author{
Selvamani Muthukalathi, Ravanan Ramanujam and Anbupalam Thalamuthu
}

\begin{abstract}
Cluster analysis in microarray gene expression studies is used to find groups of correlated and co-regulated genes. Several clustering algorithms are available in the literature. However no single algorithm is optimal for data generated under different technological platforms and experimental conditions. It is possible to combine several clustering methods and solutions using an ensemble approach. The method also known as consensus clustering is used here to examine the robustness of cluster solutions from several different algorithms. The method proposed here also is useful for estimating the number of clusters in a dataset. Here we examine the properties of consensus clustering using real and simulated datasets.
\end{abstract}

\section{INTRODUCTION}

$\mathrm{O}$ $\mathrm{NE}$ of the fundamental issues in microarray analysis is to identify the groups of co-regulated genes, which is done using the cluster analysis techniques. Several different cluster algorithms for microarray gene expression studies are available in the literature (see for example Jiang et al 2004). Performance of any particular method depends on the nature of the data and level of noise present in the dataset. To improve the stability of the cluster solutions, clusters from several different algorithms can be combined. This type of approach is described in different terminologies like, consensus, ensembles, meta-clustering and cluster aggregation etc. Here we focus an approach proposed by Monti et al (2003) and is implanted in an R package called clusterCons (Simpson et al. 2010). This package proves an approach to estimate the number of clusters and an ability to assess the robustness of the cluster solution.

Here we combine the solutions from several clustering algorithms using the consensus matrix. We also introduce a novel method to construct the cluster solution from the consensus matrix based on an approach similar to tight clustering (Tseng et al. 2005). Our method depends only on a single tuning parameter and will automatically produce an estimate of the number of clusters. The method can be implemented using a single clustering algorithm with data resampling and multiple runs. It is also possible to use multiple clustering algorithms together with the resampling techniques.

Selvamani Muthukalathi, Department of Statistics, Asan Memorial College, Chennai- 600100

Ravanan Ramanujam, Department of Statistics, Presidency College, Chennai-600 005

Anbupalam Thalamuthu, Centre for Healthy Brain Ageing, School of Psychiatry, University of New South Wales, Sydney, Australia
We have evaluated the performance of our approach using extensive simulation and also using several different real data sets.

\section{Methodology}

Let $\mathrm{X}$ denotes the $\mathrm{n} \mathrm{x} d$ microarray data matrix, with rows corresponding to the genes and columns corresponding sample. Further, we also assume that the data matrix is preprocessed and normalized (Tseng et al 2001). The purpose of cluster analysis is to find a non-overlapping partition of the data matrix such that within a group or cluster the genes are similar in some sense and between the two groups the genes are dissimilar. For example we can write the cluster solution as a $K$-cluster partition $\boldsymbol{P}$ of $X, P=\left\{P_{1}, P_{\left.2, \ldots, P_{K}\right\}}\right.$ such that $\bigcup_{k=1}^{K} P_{k}=X$ and $P_{i} \cap P_{j}=\phi, \forall i, j: i \neq j$. The number of genes within a cluster, $P_{i}$ is $n_{i}$ and $\sum_{i=1}^{K} n_{i}=n$. The number of clusters $K$ is unknown, however we assume that it is known in advance. Estimation of the number of clusters within a dataset is a very challenging problem.

Several indices to evaluate the performance of a cluster solution exist in the literature. Milligan and Cooper (1985) evaluated more than 30 indices in relation to the estimation of number of clusters and found that none is optimal for all types of data. Also there are various methods proposed in the literature for estimating K (Walther and Hastie 2000, Tibshirani et al. 2001, Dudoit and Fridlyand 2002). Here we outline a method to estimate $K$ using a tuning parameter.

\section{A. Connectivity and Consensus Matrices}

First we describe the connectivity matrix based on resampling approach. Let $X^{(1)}, X^{(2)}, \ldots, X^{(H)}$ denote the list of $H$ perturbed datasets obtained by resampling the original dataset $X$. The connectivity matrix $C$ for a cluster solution an algorithm can be defined as below:

$$
C^{(h)}(i, j)=\left\{\begin{array}{l}
1 \text { if the genes } i \text { and } j \text { appear in the same cluster } \\
0 \text { otherwise }
\end{array}\right.
$$

For resampled data $X^{(h)}$, let $I^{(h)}$ denote the indicator matrix such that its $(i, j)$-th entry is equal to 1 if both the genes $i$ and $j$ are selected in $X^{(h)}$. The combined connectivity matrix, known as the consensus matrix $M$, for all the resampled data, is defined as, $M(i, j)=\frac{\sum_{h} C^{(h)}(i, j)}{\sum_{h} I^{(h)}(i, j)}$. 
The $(\mathrm{i}, \mathrm{j})$ th entry in the consensus gives the proportion of times the pairs $(\mathrm{i}, \mathrm{j})$ appeared in the same cluster together out of the total number of times they both were selected in the resampling.

It should be noted that the consensus matrix gives a measure of similarity between two genes and hence I-M, I is the identity matrix, can be defined as a distance matrix. This distance matrix can be used in all the clustering algorithms, which uses distance matrix as an input. For example, Monte et al. [3] use the properties of the consensus matrix to identify the best cluster solution based on several clustering algorithms and re-sampled data sets.

\begin{tabular}{|c|c|c|c|c|}
\hline & $\mathrm{a}$ & b & c & d \\
\hline$c$ & 1 & 1 & O & O \\
\hline$t$ & 1 & 1 & 0.57 & 0.17 \\
\hline$c$ & O & 0.57 & 1 & 0.71 \\
\hline c & O & 0.17 & 0.71 & 1 \\
\hline$M=\epsilon$ & O & 0.14 & 0.57 & 1 \\
\hline 1 & O & O & 0.11 & 0.43 \\
\hline$c$ & 1 & 1 & 0.38 & 0.14 \\
\hline$r$ & O & 0.17 & O & O \\
\hline$i$ & 1 & 1 & 0.29 & O \\
\hline
\end{tabular}

If we set the parameter $\delta=0.7$, and group the genes which appear together in the consensus matrix above, we can obtain the three cluster solution, cluster $1=\{a, b, g, i\}$, cluster $2=$ $\{c, d, e\}$ and cluster $3=\{f, h\}$. Note that we club together all those genes that are not part of the any cluster into one cluster and call it a "noise" cluster. In this example the genes $f$ and $h$ do not cluster with any other genes and therefore they form a "noise" cluster.

The tuning parameter induces a cluster solution and provides an estimate of the number of clusters. A large value of delta will result in large number of cluster and a very small value will put all the genes in the few clusters. If the data is very noisy we may have to choose a liberal value for the tuning parameter $\delta$. We also note that if there are a large number of clusters with very few observations within each cluster then we may also combine all these isolated clusters into a single "noise" cluster.

\section{Rand and Modified Rand Indices}

For the simulated data sets we know the cluster labels and hence the cluster solutions can be compared with the original cluster labels. An index for evaluating the concordance of the original cluster label and the estimated cluster labels by the clustering method is the Rand index (Rand 1971 ; Huber and Arabie (1985). For cluster solution with a noise cluster, the Rand index was modified by Thalamuthu et al (2006). Here we describe the modified rand index as presented in Thalamuthu et al (2006).

Let $P_{R}\left(X, C_{R}\right)$ be the true partition and $P_{C}\left(X, C_{C}\right)$ be the cluster solution obtained from a clustering algorithm. Let the group labels for these two partitions respectively be $C_{R}=\left\{u_{1}\right.$, $\left.\mathrm{u}_{2}, \ldots, \mathrm{u}_{\mathrm{R}}, \mathrm{u}_{\mathrm{R}+1}\right\}$ and $\mathrm{C}_{\mathrm{C}}=\left\{\mathrm{v}_{1}, \mathrm{v}_{2}, \ldots, \mathrm{v}_{\mathrm{C}}, \mathrm{v}_{\mathrm{C}+1}\right\}$ where $\mathrm{u}_{\mathrm{R}+1}$ and $\mathrm{v}_{\mathrm{C}+1}$ are the scattered gene sets. The cross tabulation of the

\section{B. Consensus Clustering}

We propose to directly use the consensus matrix to obtain a cluster solution instead of using this matrix as an input data for another clustering algorithm. Suppose we set a threshold on the proportion of times, the pairs of genes appear together among the set of cluster solutions then it is possible to obtain a cluster solution based on this threshold parameter $\delta$.

For example assume that we have obtained the following consensus matrix for the set of 9 genes $\{\mathrm{a}, \mathrm{b}, \mathrm{c}, \ldots, \mathrm{i}\}$ as below:

$\left.\begin{array}{lllll}\mathrm{e} & \mathrm{f} & \mathrm{g} & \mathrm{h} & \mathrm{i} \\ \mathrm{O} & \mathrm{O} & 1 & \mathrm{O} & 1 \\ 0.14 & \mathrm{O} & 1 & 0.17 & 1 \\ 0.57 & 0.11 & 0.38 & \mathrm{O} & 0.29 \\ 1 & 0.43 & 0.14 & \mathrm{O} & \mathrm{O} \\ 1 & 0.43 & 0.14 & \mathrm{O} & \mathrm{O} \\ 0.43 & 1 & 0 & 0 & 0 \\ 0.14 & 0 & 1 & 0.17 & 1 \\ 0 & 0 & 0.17 & 1 & 0.2 \\ 0 & 0 & 1 & 0.2 & 1\end{array}\right)$

labels of two partitions can be given as in Table 1. The entry $n_{i j}$ denotes the number of genes belonging to the $i$-th group $u_{i}$ in partition $P_{R}\left(X, C_{R}\right)$ and $j$-the group $v_{j}$ in partition $P C\left(X, C_{C}\right)$. The rand index which can be defined as the proportion of concordant gene pairs in two partitions among all possible pairs of genes. This index was proposed for partitions without "noise" cluster.

\begin{tabular}{|l|l|l|l|l|l|l|}
\hline & $\mathrm{v}_{1}$ & $\mathrm{v}_{2}$ & $\ldots$ & $\mathrm{v}_{\mathrm{C}}$ & $\mathrm{v}_{\mathrm{C}+1}$ & Total \\
\hline $\mathrm{u}_{1}$ & $\mathrm{n}_{11}$ & $\mathrm{n}_{21}$ & $\ldots$ & $\mathrm{n}_{2 \mathrm{c}}$ & $\mathrm{n}_{2(\mathrm{c}+1)}$ & $\mathrm{n}_{1 .}$ \\
\hline $\mathrm{u}_{2}$ & $\mathrm{n}_{21}$ & $\mathrm{n}_{22}$ & $\ldots$ & $\mathrm{n}_{2 \mathrm{c}}$ & $\mathrm{n}_{2(\mathrm{c}+1)}$ & $\mathrm{n}_{2 .}$ \\
\hline$\ldots$ & $\ldots$ & $\ldots$ & $\ldots$ & $\ldots$ & $\ldots$ & $\ldots$ \\
\hline $\mathrm{u}_{\mathrm{R}}$ & $\mathrm{n}_{\mathrm{R} 1}$ & $\mathrm{n}_{\mathrm{R} 2}$ & $\ldots$ & $\mathrm{n}_{\mathrm{Rc}}$ & $\mathrm{n}_{\mathrm{R}(\mathrm{c}+1)}$ & $\mathrm{n}_{\mathrm{R} .}$ \\
\hline $\mathrm{u}_{\mathrm{R}+1}$ & $\mathrm{n}_{(\mathrm{R}+1) 1}$ & $\mathrm{n}_{(\mathrm{R}+1) 2}$ & $\ldots$ & $\mathrm{n}_{(\mathrm{R}+1) \mathrm{c}}$ & $\begin{array}{l}\mathrm{n}_{(\mathrm{R}+1)} \\
(\mathrm{c}+1)\end{array}$ & $\mathrm{n}_{\mathrm{R}+1 .}$ \\
\hline Total & $\mathrm{n}_{.1}$ & $\mathrm{n}_{2}$ & $\ldots$ & $\mathrm{n}_{. \mathrm{c}}$ & $\mathrm{n}_{.(\mathrm{c}+1)}$ & $\mathrm{n}_{. . .} \mathrm{n}$ \\
\hline
\end{tabular}

The standardized rand index proposed by Huber and Arabie (1985) is given by

$\operatorname{Rand}(\mathrm{R}, \mathrm{C})=\frac{\sum_{i=1}^{R} \sum_{j=1}^{c}\left(\begin{array}{c}n_{i j} \\ 2\end{array}\right)-\sum_{i=1}^{R}\left(\begin{array}{c}n_{i} \\ 2\end{array}\right) \sum_{j=1}^{c}\left(\begin{array}{c}n_{j} \\ 2\end{array}\right) /\left(\begin{array}{c}n \\ 2\end{array}\right)}{0.5\left[\sum_{i=1}^{R} \sum_{j=1}^{c}\left(\begin{array}{c}n_{i} \\ 2\end{array}\right)+\sum_{j=1}^{c}\left(\begin{array}{c}n_{j} \\ 2\end{array}\right)\right]-\sum_{i=1}^{R}\left(\begin{array}{c}n_{i} \\ 2\end{array}\right) \sum_{j=1}^{c}\left(\begin{array}{c}n_{j} \\ 2\end{array}\right) /\left(\begin{array}{c}n \\ 2\end{array}\right)}(1)$

This index can be extended for partitions with scattered gene sets as below.

$\operatorname{Rand}_{1}^{*}(\mathrm{R}, \mathrm{C})=$ $\frac{\sum_{i=1}^{R+1} \sum_{j=1}^{c+1}\left(\begin{array}{c}n_{i j} \\ 2\end{array}\right)-\sum_{i=1}^{R+1}\left(\begin{array}{c}n_{i .} \\ 2\end{array}\right) \sum_{j=1}^{c+1}\left(\begin{array}{c}n_{j} j \\ 2\end{array}\right) /\left(\begin{array}{c}n \\ 2\end{array}\right)}{0.5\left[\sum_{i=1}^{R+1} \sum_{j=1}^{c+1}\left(\begin{array}{c}n_{i .} \\ 2\end{array}\right)+\sum_{j=1}^{c}\left(\begin{array}{c}n_{j} \\ 2\end{array}\right)\right]-\sum_{i=1}^{R+1}\left(\begin{array}{c}n_{i .} \\ 2\end{array}\right) \sum_{j=1}^{c+1}\left(\begin{array}{c}n_{j} \\ 2\end{array}\right) /\left(\begin{array}{c}n \\ 2\end{array}\right)}$

However the above formula treats scattered genes with equal importance as the clustered genes in concordance evaluation and results in bias against methods without scattered genes especially when $n_{(R+1)}$. is large. Therefore a simple alternative is to ignore all scattered genes in either partitions and define the new modified Rand index only based on intersection of clustered genes of the two partitions: 


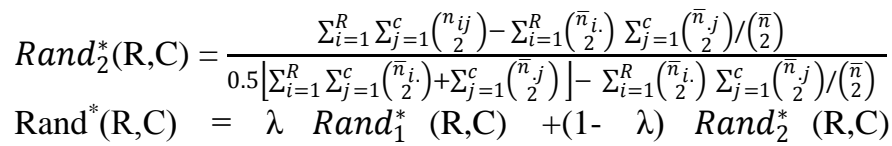
(2)

Where $\lambda=\left|\mathrm{u}_{\mathrm{R}+1} \mathrm{U} \mathrm{v}_{\mathrm{C}+1}\right| / \mathrm{n}=\mathrm{n}_{(\mathrm{R}+1)^{*}}+\mathrm{n}_{*(\mathrm{C}+1)}-\mathrm{n}_{(\mathrm{R}+1)(\mathrm{C}+1)} / \mathrm{n}$ and |.| denotes the number of genes in the gene set. Note that both $\operatorname{Rand}_{1}^{*}(\mathrm{R}, \mathrm{C})$, $\operatorname{Rand}_{2}^{*}(\mathrm{R}, \mathrm{C})$ and thus $\operatorname{Rand}^{*}(\mathrm{R}, \mathrm{C})$ take maximum value 1 when $\mathrm{P}_{\mathrm{R}}\left(\mathrm{X}, \mathrm{C}_{\mathrm{R}}\right)$ and $\mathrm{P}_{\mathrm{C}}\left(\mathrm{X}, \mathrm{C}_{\mathrm{C}}\right)$ are perfectly identical and have expected value 0 and $\mathrm{P}_{\mathrm{C}}\left(\mathrm{X}, \mathrm{C}_{\mathrm{C}}\right)$ is random partition. When $\mu_{\mathrm{R}+1}=v_{\mathrm{C}+1}=\varphi$, $\operatorname{Rand}^{*}(\mathrm{R}, \mathrm{C}) \operatorname{Rand}_{1}^{*}(\mathrm{R}, \mathrm{C})$, and

$\operatorname{Rand}_{2}^{*}(\mathrm{R}, \mathrm{C})$ all reduced to the original modified Rand index, Rand $(R, C)$ in equation(1).

\section{SimUlated DATASETS}

Thalamuthu et al (2006) have used extensive simulations to evaluate the performance of several clustering algorithms. These datasets are publically available and can be downloaded from www.biostat.pitt.edu/bioinfo/publication.htm. We have used these simulated datasets to evaluate the performance of consensus clustering with other standard methods.

Here the method of simulation used in Thalamuthu et al. (2006) is described briefly. First a template of 15 clusters of genes for 50 samples $(d=50)$ were generated. The size of each cluster $\mathrm{n}_{\mathrm{c}}(\mathrm{c}=1, \ldots, 15)$ was generated from $\mathrm{n}_{\mathrm{c}} \sim 4 \times$
Poisson(10). After the initial data was generated, the data matrix was perturbed using three perturbation models. In the first perturbation (Type I) randomly simulated genes ( $0 \%, 5 \%$, $10 \%, 20 \%, 60 \%, 100 \%, 200 \%$ ) were added to the original data matrix. Thus the Type I has 7 datasets including the original data without any scattered genes. Under Type II perturbation, an addition of a small random error from the Normal distribution with standard deviation $(\mathrm{SD})=0.05,0.1$, $0.2,0.4,0.8,1.2$ was added to each element of the gene expression matrix; a total of 6 datasets, one for each SD. The third perturbation model, Type III, involves an addition of $100 \%$ and Type IV involves addition of $200 \%$ of scattered genes to the datasets in Type II. Hence, the third and fourth perturbation models consist of 12 datasets. Including all the three perturbation models, there are a total of 25 datasets from the original gene expression dataset.

\section{REAL Microarray DatASETS}

To evaluate the performance of our clustering algorithm for real datasets we have considered the datasets used by Monti et al. (2003). These datasets are often used as benchmarks to evaluate the cluster analysis techniques for microarray data (Vukicevic et al. 2011), Table 1 shows the real datasets used and their respective number of clusters.

Table 1: Summary of Real Datasets

\begin{tabular}{|l|l|l|l|l|l|}
\hline \multicolumn{1}{|c|}{ Dataset } & \multicolumn{1}{|c|}{ Reference } & \multicolumn{1}{c|}{ Datasets } & $K$ & d & n \\
\hline Leukemia & Golub et. al (1999) & Leukemia & 3 & 38 & 999 \\
\hline Novartis & Su et. al. (2002) & Novartis & 4 & 103 & 1000 \\
\hline Lung Cancer & Bhattacharjee(2001) & Lung Cancer & 4 & 197 & 1000 \\
\hline Normal tissues & Ramaswamy et.al. (2001) & Normal tissues & 13 & 90 & 1277 \\
\hline
\end{tabular}

\section{RESUlTS AND DisCUSSION}

The standard clustering methods such as k-means, the hierarchical clustering method based on Diana algorithm, Partition Around Medoid (PAM) and model based clustering Mclust were used for comparison and calculation of consensus clustering. We have also obtained a cluster solution using the combined consensus matrix, which is the average of all the four consensus matrices of the individual methods. The Rpackage cluster (Maechler et al 2012), and mclust [Fraley and Raftery (2002)] were used for clustering. For each method, 100 bootstrap samples were used to construct the consensus matrix. For each iteration, $80 \%$ of the original samples were resampled. Since we do not know the exact number of clusters in each datasets, we have constructed the consensus matrix for a range of number of clusters together with bootstrapping. For the simulated data sets the number of clusters were varied between 5 and 25 and for real data sets it was varied between 4 and 10. The goodness of cluster solutions was examined using the rand index, modified rand index (Thalamuthu et al. 2006). The rand index is a measure of concordance between the true cluster label (for the simulated data sets we know the cluster labels and for the real datasets we have used the cluster labels as the different experimental conditions under which these data sets were generated) and the cluster label identified by any particular method. The rand index lies between 0 and 1 and a high value for this index is indicative of good concordance between the original cluster labels and the estimated cluster labels. Since the rand index gives equal weights to the genes or observations that are clustered together and the noise observations, a modified rand index which is a weighted average of the rand index with and without noise observations was proposed in (Thalamuthu et al. 2006). We have computed both the rand index (1) and the modified rand index (2) for our analysis.

The results of the simulated datasets are summarized in Tables 2 and Table 3 . The last five columns of the tables give the results for each of the four methods and the combined cluster for type. The rand index, modified rand index and the estimated number of clusters together with the $\delta$ used to obtain the cluster solution are presented here.

In general the consensus clustering approach is robust to perturbation of the observations, i.e. adding additional error component to the original gene expression (such as the Type II simulation). As expected, addition of noise observations affects the clustering algorithms to a large extent and hence the rand indices and the modified rand indices are very small. This is not a limitation of the methodologies because the noise observations are not generated as a single cluster, as assumed in the calculation of rand and modified rand indices, and the noise observations by themselves may form multiple clusters as it can be seen from the estimate of the number of clusters under these cases. It is also interesting to note that the rand 
indices and modified rand indices are in close agreement with the results obtained in Thalamuthu et al. (2006).

For the real datasets (Table 4), our estimates of the number of clusters are very close to the number of classes available in these datasets. Note that the $\delta$ values for these datasets are very small due to the fact that the sample sizes in these datasets are small. A high value for $\delta$ will split the samples into many groups and the level of noise increases, which may not be optimal, but by setting a high value for $\delta$ it is possible to identify closely related samples. In general it is a trade-off between the level of noise and the number of clusters.

The delta for which the estimated number of clusters is close to the true clusters is presented in the table 6. However other values are delta and the rand index comparing the cluster labels and the original labels of the real data sets are presented in Figures 1 to 4 . As expected, the pattern of the estimated clusters and the rand indices are not uniform across all data sets. The consensus solution obtained using hierarchical cluster (Diana) is not very much affected the delta values and hence the solution is stable across various values of delta.
We have not provided the comparison of the estimated number of clusters with other methods such as gab statistics (Tibshirani et al 2001) or the clusterCons package. We are compiling these results and will be published elsewhere. For large data sets such as our simulated datasets, the package clusterCons take very long time to run. Therefore we have implemented their approach in $\mathrm{R}$ and will soon be available with our approach as a package. Further, we have not provided a clear guidance for setting the parameter $\delta$. In general, we should have some idea about the number of clusters and level of noise in the data. But the distribution of the elements in the consensus matrix will help us to decide the delta. For large data sets such as a typical microarray dataset a higher $\delta$ will be able identify set of closely co-regulated genes. For smaller data sets with few sample points as in classification problem with microarrays we would use a small $\delta$ value. We are working on an approach to suggest a $\delta$ value based on the distribution of cluster similarities.

Table 2: Summary of Results for Type I and Type II Simulations

\begin{tabular}{|c|c|c|c|c|c|c|c|c|c|c|}
\hline & \multicolumn{5}{|c|}{ Type I } & \multicolumn{5}{|c|}{ Type II } \\
\hline & combined & Diana & kmean & Mclus & Pam & combined & Diana & kmean & Mclust & Pam \\
\hline Parameter & \multicolumn{5}{|c|}{$\%$ Noise $=0 ; \mathrm{SD}=0 ;$ delta $=0.5725 ;$ Ncluster $=15$} & \multicolumn{5}{|c|}{$\%$ Noise $=0 ;$ SD $=0 ;$ delta $=0.5625 ;$ Ncluster $=15$} \\
\hline Rand & 1 & 1 & 1 & 1 & 1 & 1 & 1 & 1 & 1 & 1 \\
\hline Mod Rand & 1 & 1 & 1 & 1 & 1 & 1 & 1 & 1 & 1 & 1 \\
\hline $\mathrm{N}$ clusters & 15 & 15 & 15 & 16 & 16 & 15 & 15 & 15 & 16 & 16 \\
\hline Parameter & \multicolumn{5}{|c|}{$\%$ Noise $=5 ;$ SD $=0 ;$ delta $=0.7125 ;$ Ncluster $=16$} & \multicolumn{5}{|c|}{ \%Noise $=0 ;$ SD $=0.05 ;$ delta $=0.5300 ;$ Ncluster=16 } \\
\hline Rand & 1 & 0.346 & 0.944 & 0.997 & 0.943 & 1 & 1 & 1 & 1 & 1 \\
\hline Mod Rand & 1 & 0.805 & 1 & 0.949 & 0.896 & 1 & 1 & 1 & 1 & 1 \\
\hline $\mathrm{N}$ clusters & 16 & 7 & 17 & 17 & 18 & 15 & 15 & 15 & 15 & 15 \\
\hline Parameter & \multicolumn{5}{|c|}{$\%$ Noise $=10 ; \mathrm{SD}=0 ;$ delta $=0.7440 ;$ Ncluster $=16$} & \multicolumn{5}{|c|}{ \%Noise $=0 ;$ SD $=0.1 ;$ delta $=0.4450 ;$ Ncluster $=16$} \\
\hline Rand & 1 & 0.349 & 0.955 & 0.992 & 0.898 & 0.921 & 0.85 & 0.921 & 1 & 0.921 \\
\hline Mod Rand & 1 & 0.799 & 0.977 & 0.999 & 0.993 & 0.972 & 0.979 & 0.993 & 1 & 0.973 \\
\hline $\mathrm{N}$ clusters & 16 & 6 & 19 & 17 & 17 & 15 & 15 & 15 & 15 & 15 \\
\hline Parameter & \multicolumn{5}{|c|}{$\%$ Noise $=20 ;$ SD $=0 ;$ delta $=0.6860 ;$ Ncluster $=16$} & \multicolumn{5}{|c|}{ \%Noise $=0 ;$ SD $=0.2 ;$ delta $=0.5700 ;$ Ncluster $=16$} \\
\hline Rand & 1 & 0.401 & 0.93 & 0.969 & 0.747 & 1 & 0.989 & 1 & 1 & 0.992 \\
\hline Mod Rand & 1 & 0.815 & 0.995 & 0.998 & 0.98 & 1 & 1 & 1 & 1 & 0.999 \\
\hline N clusters & 16 & 7 & 19 & 18 & 19 & 15 & 16 & 15 & 15 & 16 \\
\hline Parameter & \multicolumn{5}{|c|}{$\%$ Noise $=60 ;$ SD $=0 ;$ delta $=0.3600 ;$ Ncluster $=16$} & \multicolumn{5}{|c|}{$\%$ Noise $=0 ;$ SD $=0.4 ;$ delta $=0.3833 ;$ Ncluster $=16$} \\
\hline Rand & 0.373 & 0.066 & 0.265 & 0.828 & 0.346 & 0.852 & 0.789 & 0.851 & 0.852 & 0.953 \\
\hline Mod Rand & 0.879 & 0.646 & 0.87 & 0.959 & 0.894 & 0.98 & 0.968 & 0.979 & 0.98 & 0.995 \\
\hline N clusters & 15 & 16 & 25 & 13 & 19 & 13 & 13 & 13 & 13 & 15 \\
\hline Parameter & \multicolumn{5}{|c|}{$\%$ Noise $=100 ;$ SD $=0 ;$ delta $=0.3200 ;$ Ncluster $=16$} & \multicolumn{5}{|c|}{ \%Noise $=0 ;$ SD $=0.8 ;$ delta $=0.3200 ;$ Ncluster $=16$} \\
\hline Rand & 0.362 & 0.025 & 0.206 & 0.512 & 0.269 & 0.581 & 0.668 & 0.835 & 0.526 & 0.62 \\
\hline Mod Rand & 0.802 & 0.704 & 0.793 & 0.851 & 0.815 & 0.923 & 0.942 & 0.977 & 0.893 & 0.943 \\
\hline N clusters & 10 & 26 & 22 & 12 & 16 & 10 & 13 & 13 & 11 & 14 \\
\hline Parameter & \multicolumn{5}{|c|}{$\%$ Noise $=200 ;$ SD $=0 ;$ delta $=0.5000 ;$ Ncluster $=16$} & \multicolumn{5}{|c|}{$\%$ Noise $=0 ; \mathrm{SD}=1.2 ;$ delta $=0.2400 ;$ Ncluster $=16$} \\
\hline Rand & .06 & .215 & .36 & .972 & .057 & 0.373 & 0.475 & 0.664 & 0.298 & 0.376 \\
\hline Mod Rand & .0 .512 & 0.648 & 0.72 & 0.988 & 0.61 & 0.835 & 0.897 & 0.928 & 0.78 & 0.893 \\
\hline $\mathrm{N}$ clusters & 49 & 55 & 52 & 15 & 48 & 7 & 11 & 11 & 6 & 13 \\
\hline
\end{tabular}


Table 3: Summary of Results for Type III Simulations (100\% noise) and Type IV Simulation (200\% Noise)

\begin{tabular}{|c|c|c|c|c|c|c|c|c|c|c|}
\hline & \multicolumn{5}{|c|}{ Type III Simulations } & \multicolumn{5}{|c|}{ Type IV Simulation } \\
\hline & Combined & Diana & kmeans & Mclust & Pam & Combined & Diana & kmeans & Mclust & Pam \\
\hline Parameter & \multicolumn{5}{|c|}{$\mathrm{SD}=0 ;$ delta $=0.32 ; \quad \mathrm{N}$ clusters $=16$} & \multicolumn{5}{|c|}{$\mathrm{SD}=0 ;$ delta $=0.50 ; \quad \mathrm{N}$ clusters $=16$} \\
\hline Rand & 0.362 & 0.025 & 0.206 & 0.512 & 0.269 & 0.06 & 0.205 & 0.365 & 0.972 & 0.057 \\
\hline Mod Rand & 0.802 & 0.704 & 0.793 & 0.851 & 0.815 & 0.612 & 0.648 & 0.724 & 0.988 & 0.61 \\
\hline N clusters & 10 & 26 & 22 & 12 & 16 & 49 & 55 & 52 & 15 & 48 \\
\hline Parameter & \multicolumn{5}{|c|}{$\mathrm{SD}=0.05 ;$ delta $=0.34 ; \quad \mathrm{N}$ clusters $=16$} & \multicolumn{5}{|c|}{$\mathrm{SD}=0.05 ;$ delta $=0.60 ; \quad \mathrm{N}$ clusters $=16$} \\
\hline Rand & 0.493 & 0.031 & 0.237 & 0.63 & 0.221 & 0.25 & 0.589 & 0.817 & 0.991 & 0.086 \\
\hline Mod Rand & 0.822 & 0.705 & 0.798 & 0.882 & 0.811 & 0.685 & 0.811 & 0.916 & 0.996 & 0.623 \\
\hline N clusters & 8 & 28 & 19 & 12 & 17 & 67 & 25 & 24 & 17 & 50 \\
\hline Parameter & \multicolumn{5}{|c|}{$\mathrm{SD}=0.1 ;$ delta $=0.20 ; \quad \mathrm{N}$ clusters $=16$} & \multicolumn{5}{|c|}{$\mathrm{SD}=0.1 ;$ delta $=0.48 ; \quad \mathrm{N}$ clusters $=16$} \\
\hline Rand & 0.308 & 0.042 & 0.5 & 0.709 & 0.302 & 0.061 & 0.175 & 0.613 & 0.977 & 0.054 \\
\hline Mod Rand & 0.721 & 0.68 & 0.828 & 0.905 & 0.808 & 0.614 & 0.647 & 0.704 & 0.996 & 0.611 \\
\hline N clusters & 4 & 12 & 8 & 9 & 12 & 50 & 47 & 60 & 15 & 48 \\
\hline Parameter & \multicolumn{5}{|c|}{$\mathrm{SD}=0.2 ;$ delta $=0.32 ; \quad \mathrm{N}$ clusters $=16$} & \multicolumn{5}{|c|}{$\mathrm{SD}=0.2 ;$ delta $=0.40 ; \quad \mathrm{N}$ clusters $=16$} \\
\hline Rand & 0.802 & 0.055 & 0.185 & 0.853 & 0.244 & 0.174 & 0.0108 & 0.036 & 0.625 & 0.049 \\
\hline Mod Rand & 0.832 & 0.707 & 0.79 & 0.944 & 0.805 & 0.65 & 0.581 & 0.597 & 0.835 & 0.609 \\
\hline N clusters & 11 & 23 & 32 & 11 & 19 & 22 & 38 & 62 & 15 & 37 \\
\hline Parameter & \multicolumn{5}{|c|}{$\mathrm{SD}=0.4 ; \quad$ delta $=0.34 ; \quad \mathrm{N}$ clusters $=16$} & \multicolumn{5}{|c|}{$\mathrm{SD}=0.4 ;$ delta $=0.32 ; \quad \mathrm{N}$ clusters $=16$} \\
\hline Rand & 0.405 & 0.002 & 0.247 & 0.726 & 0.244 & 0.249 & 0.006 & 0.063 & 0.935 & 0.111 \\
\hline Mod Rand & 0.82 & 0.695 & 0.81 & 0.911 & 0.805 & 0.671 & 0.576 & 0.611 & 0.968 & 0.628 \\
\hline N clusters & 11 & 25 & 21 & 12 & 20 & 13 & 23 & 34 & 12 & 21 \\
\hline Parameter & \multicolumn{5}{|c|}{$\mathrm{SD}=0.8 ;$ delta $=0.24 ; \quad \mathrm{N}$ clusters $=16$} & \multicolumn{5}{|c|}{$\mathrm{SD}=0.8 ;$ delta $=0.20 ; \quad \mathrm{N}$ clusters $=16$} \\
\hline Rand & 0.28 & 0.08 & 0.375 & 0.422 & 0.095 & 0.139 & 0.073 & 0.183 & 0.547 & 0.085 \\
\hline Mod Rand & 0.78 & 0.731 & 0.815 & 0.722 & 0.764 & 0.616 & 0.6 & 0.651 & 0.766 & 0.606 \\
\hline N clusters & 10 & 11 & 13 & 5 & 16 & 9 & 12 & 17 & 5 & 13 \\
\hline Parameter & \multicolumn{5}{|c|}{$\mathrm{SD}=1.2 ; \quad$ delta $=0.22 ; \quad \mathrm{N}$ clusters $=16$} & \multicolumn{5}{|c|}{$\mathrm{SD}=1.2 ;$ delta $=0.50 ; \quad \mathrm{N}$ clusters $=16$} \\
\hline Rand & 0.326 & 0.074 & 0.1664 & 0.303 & 0.084 & 0.056 & 0.094 & 0.592 & 0.29 & 0.0183 \\
\hline Mod Rand & 0.776 & 0.725 & 0.777 & 0.704 & 0.744 & 0.603 & 0.615 & 0.81 & 0.665 & 0.583 \\
\hline $\mathrm{N}$ clusters & 8 & 11 & 17 & 8 & 15 & 87 & 66 & 35 & 10 & 77 \\
\hline
\end{tabular}

Table 4: Summary of Results for Real Datasets

\begin{tabular}{|l|l|l|l|l|l|l|l|l|}
\hline Data sets & & N True Clusters & Delta & Combined & kmeans & Mclust & Pam & Diana \\
\hline St. jude Leukemia & & 6 & 0.280 & & & & & \\
\hline & Rand & & & 0.918 & 0.945 & 0.946 & 0.918 & 0.457 \\
\hline & Mod Rand & & & 0.958 & 0.974 & 0.971 & 0.974 & 0.786 \\
\hline & N clusters & & & 7 & 6 & 7 & 6 & 6 \\
\hline & & 4 & 0.200 & & & & & \\
\hline & Rand & & & 0.553 & 0.426 & 0.395 & 0.375 & 0.544 \\
\hline & Mod Rand & & & 0.813 & 0.732 & 0.744 & 0.650 & 0.751 \\
\hline & N clusters & & & 5 & 6 & 4 & 5 & 4 \\
\hline Normal Tissues Cancer & & 13 & 0.260 & & & & & \\
\hline & Rand & & & 0.306 & 0.301 & 0.233 & 0.373 & 0.213 \\
\hline & Mod Rand & & & 0.803 & 0.839 & 0.767 & 0.858 & 0.744 \\
\hline & N clusters & & & 5 & 7 & 6 & 7 & 6 \\
\hline
\end{tabular}




\begin{tabular}{|c|c|c|c|c|c|c|c|c|}
\hline & Rand & & & 0.588 & 0.527 & 0.742 & 0.496 & 0.516 \\
\hline & Mod Rand & & & 0.823 & 0.822 & 0.902 & 0.792 & 0.764 \\
\hline & $\mathrm{N}$ clusters & & & 4 & 4 & 3 & 5 & 4 \\
\hline \multirow[t]{4}{*}{ CNS Tumors } & & 5 & 0.300 & & & & & \\
\hline & Rand & & & 0.443 & 0.368 & 0.525 & 0.581 & 0.311 \\
\hline & Mod Rand & & & 0.839 & 0.813 & 0.856 & 0.894 & 0.667 \\
\hline & N clusters & & & 5 & 5 & 5 & 5 & 3 \\
\hline \multirow[t]{4}{*}{ Novartis } & & 4 & 0.260 & & & & & \\
\hline & Rand & & & 0.89 & 0.881 & 0.890 & 0.862 & 0.778 \\
\hline & Mod Rand & & & 0.972 & 0.953 & 0.972 & 0.95 & 0.927 \\
\hline & $\mathrm{N}$ clusters & & & 5 & 6 & 6 & 7 & 6 \\
\hline
\end{tabular}

In the following Figures 1 to 4 , rand indices and the estimated number of clusters based on consensus cluster obtained using different algorithms and the combined consensus algorithm for the real data. Rand index for various values of delta are plotted and the estimated number of clusters at each combination of parameters is presented within the rectangles

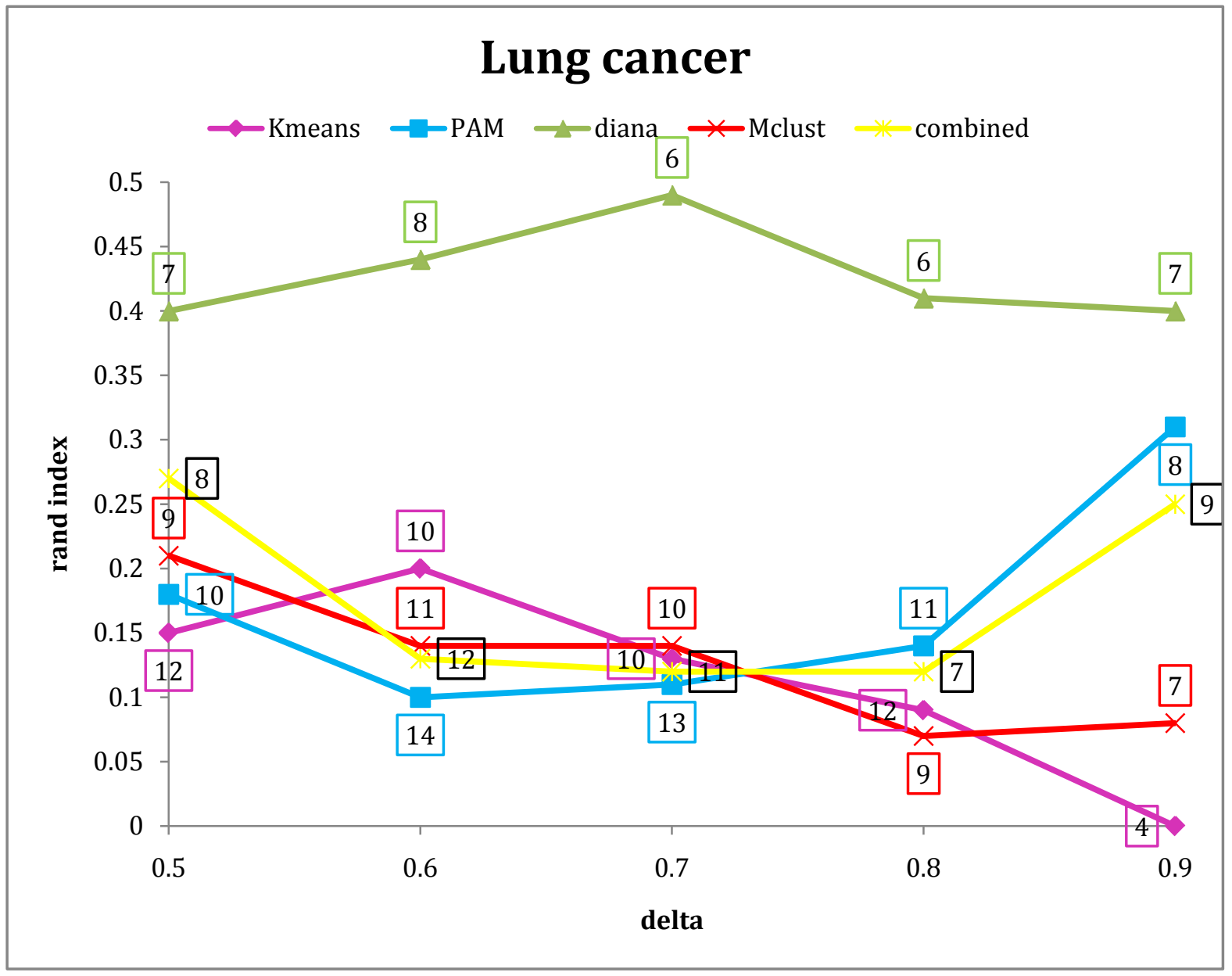

Figure 1 


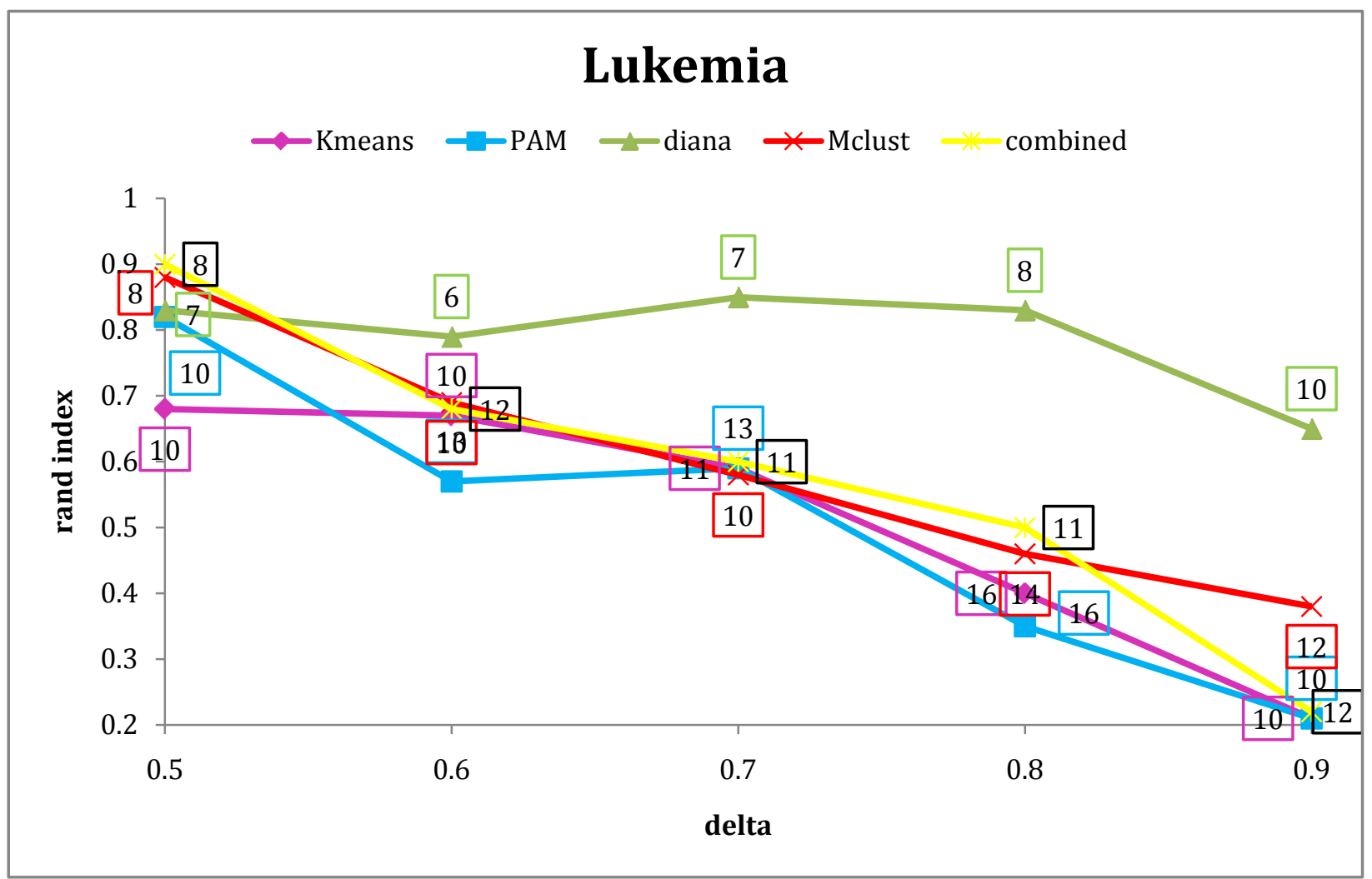

Figure 2

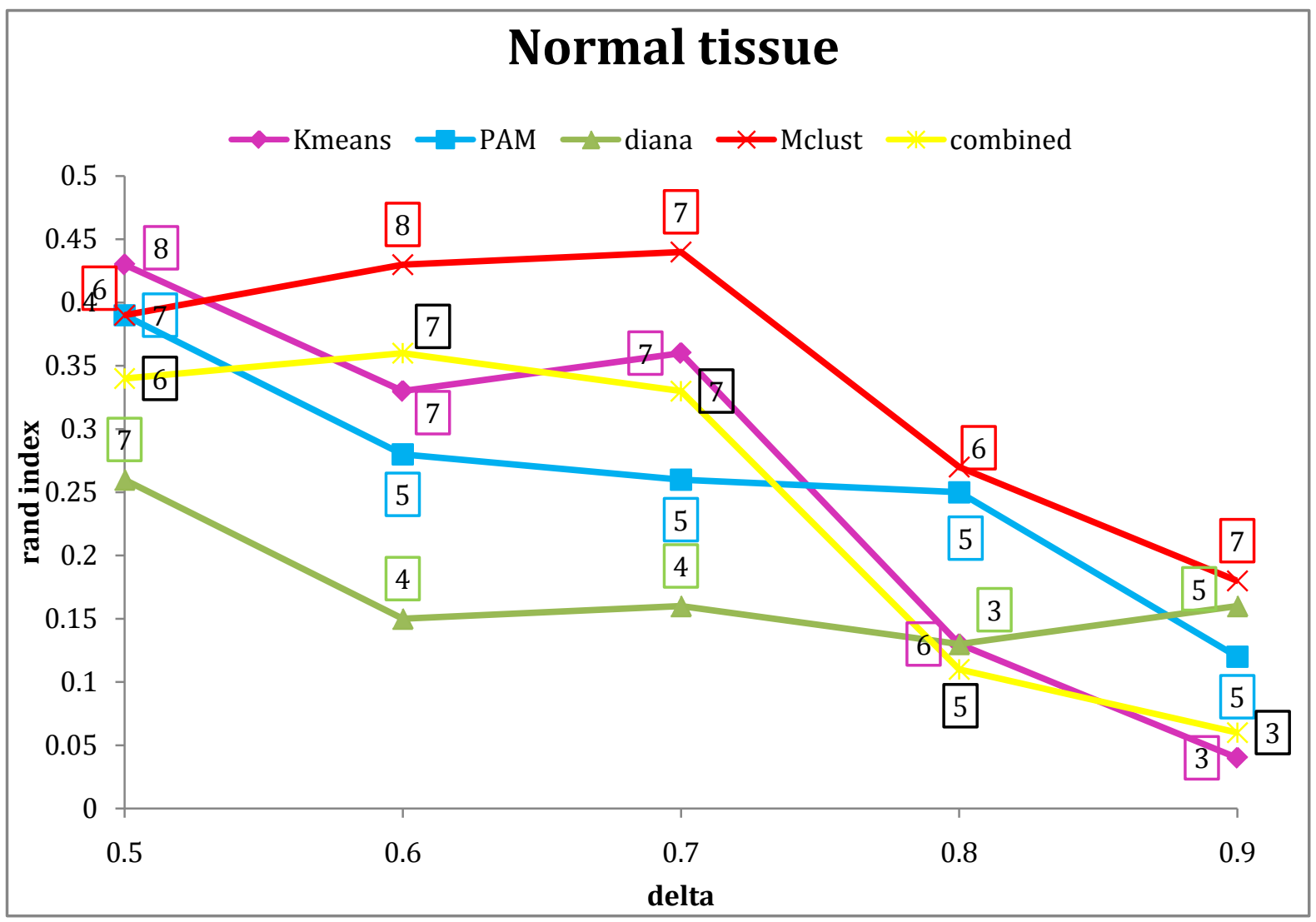

Figure 3 


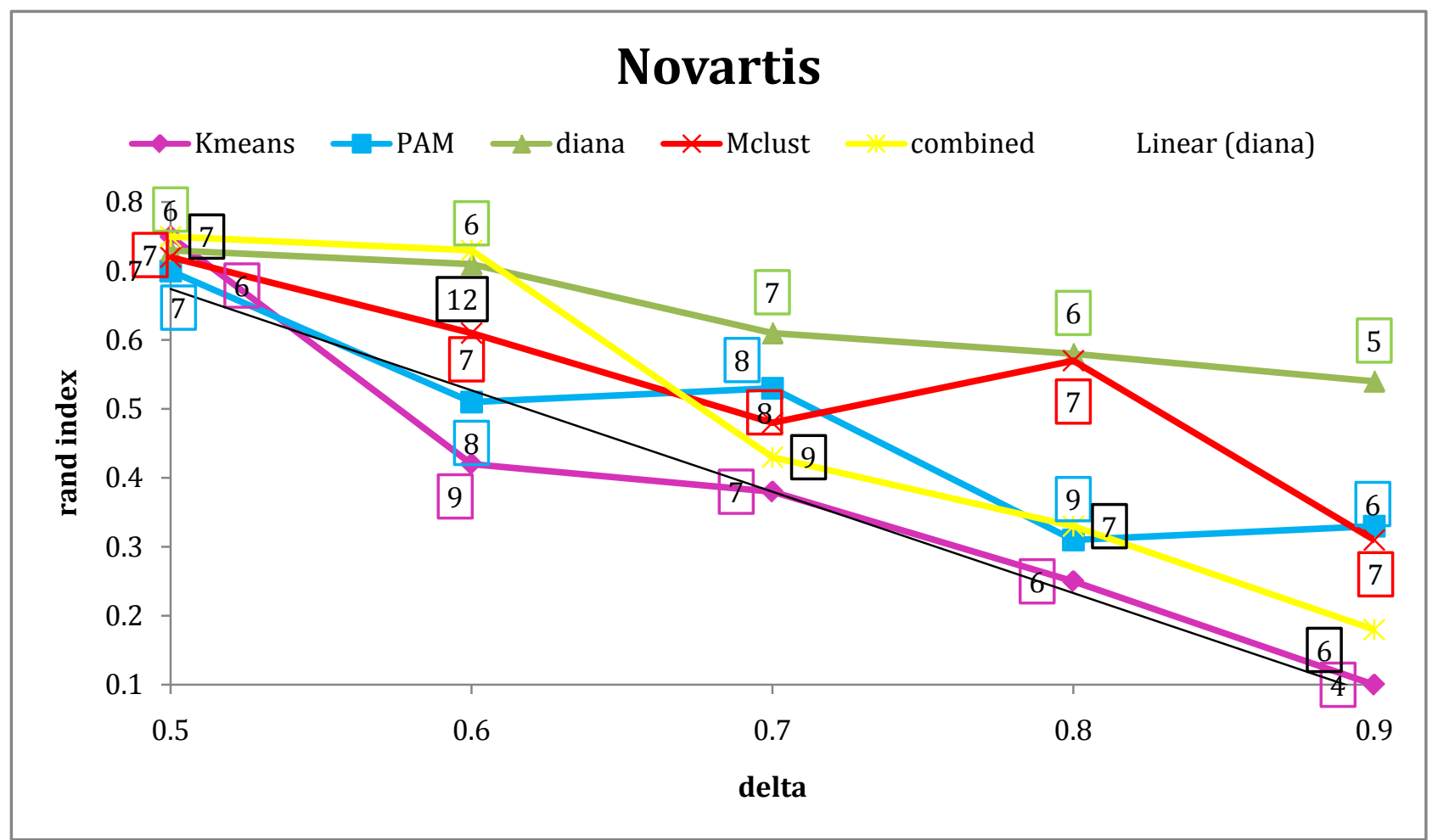

Figure 4

\section{REFERENCES}

[1] Bhattacharjee, A., W. G. Richards, J. Staunton, C. Li, S. Monti, P. Vasa, C. Ladd, J. Beheshti, R. Bueno, M. Gillette, M. Loda, G. Weber, E. J. Mark, E. S. Lander, W. Wong, B. E. Johnson, T. R. Golub, D. J. Sugarbaker, and M. Meyerson: 2001, 'Classification of Human Lung Carcinomas by mRNA Expression Profiling Reveals Distinct Adenocarcinomas Sub-classes'. Proceedings of the National Academy of Sciences 98(24), 13790-13795

[2] Duboit. S, and Frdlyland. J .(2002) "A Prediction based Resampling methods for estimating the number of clusters in a data set”, Genome Biology 3(7), 1-21.

[3] Fraley.C and Raftery.A.E, (2002) Model-based Clustering, Discriminant Analysis and Density Estimation Journal of the American Statistical Association 97:611-631

[4] Fraley.C, Raftery.A.E, T. Brendan Murphy, and Luca Scrucca (2012) mclust Version 4 for R: Normal Mixture Modeling for Model-Based Clustering, Classification, and Density Estimation Technical Report No. 597, Department of Statistics, University of Washington

[5] Golub, T. R., D. K. Slonim, P. Tamayo, C. Huard, M. Gaasenbeek, J. P.Mesirov, H. Coller, M. Loh, J. Downing, M. Caligiuri, C. Bloomfield, , and E.Lander: 1999, 'Molecular Classification of Cancer: Class Discovery and ClassPrediction by Gene Expression'. Science 286(5439), 531-537.

[6] Hubert,J. and Arabie,P. (1985) Comparing partitions. J. Classif., 2, 193218.

[7] Jiang.D, Tang. C., Jang.A. (2004). Cluster analysis for gene expression data: a survey IEE Transactions on Knowledge and Data Engineering. Vol. 16 (11), $1370-1386$.

[8] Maechler, M., Rousseeuw, P., Struyf, A., Hubert, M., Hornik, K.(2012). cluster: Cluster Analysis Basics and Extensions. R package version 1.14.2.

[9] Milligan. G.W., and Cooper.M.C.,, (1985 ) “An examination of procedures for determining the number of clusters in a data set," Psychometrika, vol. 50, no. 2, pp. 159-179

[10] Monti, S., et al., (2003) Consensus Clustering: A Resampling-Based Method for Class Discovery and Visualization of Gene Expression Microarray Data. Machine Learning, 52(1): p. 91-118.

[11] Ramaswamy, S., P. Tamayo, R. Rifkin, S. Mukherjee, C.-H. Yeang, M. Angelo, C. Ladd, M. Reich, E. Latulippe, J. P. Mesirov, T. Poggio, W.
Gerald, M. Loda, E. S. Lander, and T. R. Golub: 2001, 'Multi-Class Cancer Diagnosis Using Tumor Gene Expression Signatures'. Proceedings of the National Academy of Sciences 98(26), 15149-15154.

[12] Rand,W.M. (1971) Objective criteria for the evaluation of clustering methods. J. Am.Stat. Assoc., 66, 846-856.

[13] Simpson, T.I., J.D. Armstrong, and A. Jarman, (2010) Merged consensus clustering to assess and improve class discovery with microarray data. BMC Bioinformatics,. 11(1): p. 590.

[14] Su, A. I., M. P. Cooke, K. A. Ching, Y. Hakak, J. R. Walker, T. Wiltshire, A. P. Orth, R. G. Vega, L. M. Sapinoso, A. Moqrich, A. Patapoutian, G. M. Hampton, P. G. Schultz, and J. B. Hogenesch: 2002, 'Large-scale Analysis of the Human and Mouse Transcriptomes'. Proceedings of the National Academy of Sciences 99(7), 4465-447.

[15] Thalamuthu.A.,, Mukhopadhyay.I.,, Zheng.X, and Tseng.G.C., (2006) "Evaluation and comparison of gene clustering methods in microarray analysis,” Bioinformatics, 22, 2405-2412

[16] Tibshirani. R., Walther. G., and Hastie.T., (2001) "Estimating the number of clusters in a dataset via the gap statistic,” Journal of the Royal Statistical Society -Series B: Statistical Methodology, 63, 411-423.

[17] Tseng,G.C. and Wong,W.H. (2005) Tight clustering: a resampling-based approach for identifying stable and tight patterns in data. Biometrics, 61, $10-16$.

[18] Tseng,G.C., Oh,M.K., Rohlin,L., Liao,J.C. and Wong,W.H. (2001) Issues in cDNA microarray analysis: quality filtering, channel normalization, models of variations and assessment of gene effects. Nucleic Acids Res., 29, 2549-2557

[19] Vukicevic. M, Delibaisc.B.,, Jovanovic. M, Suknovic.M and Obradovic. Z., (2011) "International evaluation measures as proxies for external gene expression microarray data", Bioinformatics and Biomedicine(BIBM) IEEE International Conference, PP 574, 577 